\title{
SPARSE MATRIX FACTORIZATIONS OF TRANSFER MATRICES
}

\author{
MURRAY T. BATCHELOR ${ }^{1}$
}

(Received 13 March, 1986)

\begin{abstract}
Sparse matrix factorizations of transfer matrices for the interactions round a face model are reviewed. The sparse factors of a more general Ising model containing first, second and third nearest neighbour interactions are also presented. For both models the factorizations are achieved by considering the required auxiliary spin sets as a hierarchy of interacting spins.
\end{abstract}

\section{Introduction}

The study of lattice models in statistical mechanics has grown at an unprecedented rate since the introduction of the transfer matrix by Kramers and Wannier [8] some 45 years ago. In one dimension, each application of the transfer matrix can be thought of as representing a transfer to the next spin along the chain. Similarly in two dimensions each application adds a row to the lattice while in three dimensions the transfer matrix builds the system up plane by plane.

The transfer matrix formalism has led to the exact solution of several two-dimensional lattice models [4]. In the absence of exact solutions however, the transfer matrix formalism can still be applied to yield numerical results. Arguably the most powerful numerical method in two dimensions is the application of phenomenological renormalization to finite lattices which are infinite in one direction but finite in the other direction, i.e. to a strip of width $n$. Interest is focussed on the leading eigenvalues $\left(\lambda_{0}>\lambda_{1} \geqslant \ldots\right)$ of the transfer matrix in terms of which the free energy per site $f_{n}$ and the correlation length $\xi_{n}$ are

\footnotetext{
${ }^{1}$ Department of Mathematics, Faculty of Science, Australian National University, Canberra, A.C.T. 2601

(1) Copyright Australian Mathematical Society 1987, Serial-fee code 0334-2700/87
} 
defined by [4]

$$
\begin{aligned}
& f_{n}=-\frac{1}{n} \ln \left(\lambda_{0}\right), \\
& \xi_{n}^{-1}=\ln \left(\lambda_{0} / \lambda_{1}\right) .
\end{aligned}
$$

Given $f_{n}$ and $\xi_{n}$, finite-size scaling can then be used to extract critical exponents and map out the phase boundaries of the bulk system (infinite in both directions) (for recent reviews of this technique see Barber [1] and Nightingale [11]).

One way of easing the computational effort involved in obtaining the leading eigenvalues is to factor the transfer matrix into a product of sparse matrices. Explicit factorizations have been given in the past for the general Interactions Round a Face (IRF) Ising model [3]. Nightingale [10] considererd the row to row transfer matrix while Baxter [3] introduced a factorization of the diagonal to diagonal transfer matrix.

The IRF model contains first and second nearest neighbour interactions. It is the purpose of this paper to review the factorizations of this model and to introduce a factorization of a transfer matrix for a model containing first, second and third nearest neighbour interactions. The factorization is achieved in each case with the introduction of auxiliary sets of spin variables. For the latter model, the explicit structure for each of the sparse factors of the transfer matrix is also presented.

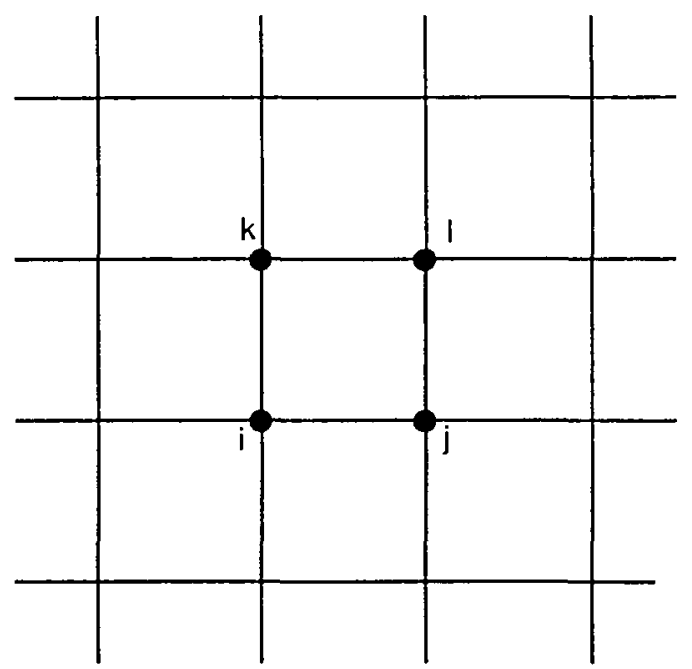

FIGURE 1 


\section{Interactions round a face}

Consider a square lattice of $N=m n$ sites where $m$ and $n$ are both even. With each site associate a spin $\sigma_{i}= \pm 1$. To each face assign a Boltzmann weight factor $w\left(\sigma_{l}, \sigma_{j}, \sigma_{k}, \sigma_{l}\right)$ where $i, j, k, l$ are the four sites round a face as in Figure 1 . The partition function is

$$
\mathbf{Z}_{N}=\sum_{\{\sigma\}} \prod_{(j, k l)} w\left(\sigma_{l}, \sigma_{\jmath}, \sigma_{k}, \sigma_{l}\right)
$$

where the summation is over all configurations of the spins and the product is over all faces of the lattice. In representing the evaluation of (2) as an eigenvalue problem we are at liberty to choose a direction in which to run a transfer matrix.

\subsection{Diagonal to diagonal transfer matrix}

With the square lattice now drawn diagonally as in Figure 2, the combined Boltzmann weight of the shaded faces is

$$
\mathbf{V}\left(\boldsymbol{\sigma} \mid \boldsymbol{\sigma}^{\prime}\right)=\prod_{j=1}^{n / 2} w\left(\sigma_{2 j-1}, \sigma_{2 j}, \sigma_{2 j+1}, \sigma_{2 j}^{\prime}\right) \delta\left(\sigma_{2 j-1}, \sigma_{2 j-1}^{\prime}\right)
$$

and for the row above

$$
\mathbf{W}\left(\boldsymbol{\sigma}^{\prime} \mid \boldsymbol{\sigma}^{\prime \prime}\right)=\prod_{j=1}^{n / 2} w\left(\boldsymbol{\sigma}_{2 j}^{\prime}, \sigma_{2 j+1}^{\prime}, \boldsymbol{\sigma}_{2 j+2}^{\prime}, \sigma_{2 j+1}^{\prime \prime}\right) \delta\left(\sigma_{2 \jmath}^{\prime}, \sigma_{2 j}^{\prime \prime}\right),
$$

where we impose periodic boundary conditions $\left(\sigma_{n+1}=\sigma_{1}\right)$. With $m$ the number of such diagonal rows, equation (2) can be written as

$$
\mathbf{Z}_{N}=\operatorname{Trace}(\mathbf{V W})^{m / 2} \text {, }
$$

where we have applied periodic boundary conditions also in the vertical direction (row $m$ is followed by row 1 ). Since each set of spins $\sigma$ on a row has $2^{n}$ configurations, $\mathrm{V}$ and $\mathrm{W}$ are both $2^{n} \times 2^{n}$ matrices.

To find factors of the matrices $\mathbf{V}$ and $\mathbf{W}$, we introduce $n-2$ auxiliary sets of spins $\mathbf{v}^{p}$ and $\mathbf{w}^{p}$ where

$$
\begin{aligned}
\mathbf{v}^{p} & =\left\{v_{1}^{p}, \ldots, v_{n}^{p}\right\}, \\
\mathbf{w}^{p} & =\left\{w_{1}^{p}, \ldots, w_{n}^{p}\right\},
\end{aligned}
$$

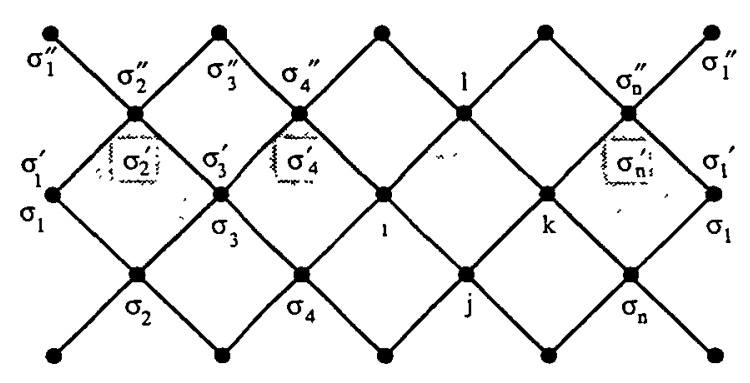

FIGURE 2 
with $p=1, \ldots, n / 2-1$. At this point it is convenient to consider Figure 3 in which the elements $V\left(\sigma \mid \sigma^{\prime}\right)$ are represented as a hierarchy of interacting spin sets $\mathbf{v}^{p}$. All spins connected with solid lines interact through delta functions. Spins connected with dashed lines interact via a Boltzmann weight factor. With Figure 2 in mind, Figure 3 can be interpreted as a schematic illustration of

$$
\mathbf{V}\left(\boldsymbol{\sigma} \mid \boldsymbol{\sigma}^{\prime}\right)=\sum_{\left\{\mathbf{v}^{D}\right\}} \mathbf{U}_{2}\left(\boldsymbol{\sigma} \mid \mathbf{v}^{1}\right) \mathbf{U}_{4}\left(\mathbf{v}^{1} \mid \mathbf{v}^{2}\right) \cdots \mathbf{U}_{n}\left(\mathbf{v}^{n / 2-1} \mid \sigma^{\prime}\right)
$$

with

$\mathbf{U}_{i}(\mathbf{s} \mid \mathbf{t})=\delta\left(s_{1}, t_{1}\right) \cdots \delta\left(s_{i-1}, t_{t-1}\right) w\left(s_{t-1}, s_{i}, s_{t+1}, t_{l}\right) \delta\left(s_{t+1}, t_{l+1}\right) \cdots \delta\left(s_{n}, t_{n}\right)$,

where $s=\left\{s_{1}, \ldots, s_{n}\right\}$ and $t=\left\{t_{1}, \ldots, t_{n}\right\}$.

Thus $\mathbf{V}$ can be written as a product of $n / 2$ matrices

$$
\mathbf{V}=\mathbf{U}_{2} \mathbf{U}_{4} \cdots \mathbf{U}_{n} \text {. }
$$

In a similar manner we write

$$
\mathbf{W}\left(\sigma^{\prime} \mid \sigma^{\prime \prime}\right)=\sum_{\left\{\mathbf{W}^{p}\right\}} \mathbf{U}_{3}\left(\sigma^{\prime} \mid \mathbf{w}^{1}\right) \mathbf{U}_{5}\left(\mathbf{w}^{1} \mid \mathbf{w}^{2}\right) \cdots \mathbf{U}_{1}\left(\mathbf{w}^{n / 2-1} \mid \sigma^{\prime \prime}\right),
$$

where the hierarchy is similar to that in Figure 3. Thus

$$
\mathbf{W}=\mathbf{U}_{3} \mathbf{U}_{5} \cdots \mathbf{U}_{1} \text {, }
$$

with $\mathbf{U}_{i}$ given by (8). Baxter [3] calls these factors face transfer matrices. It is precisely these $U_{t}$ that appear in the corner transfer matrix formalism (see e.g. [4]). From the definition (8) one can easily prove the following commutation relation

$$
\mathbf{U}_{\imath} \mathbf{U}_{j}=\mathbf{U}_{j} \mathbf{U}_{\imath} \text { for }|i-j|>1 \text {. }
$$

Thus the order in (9) and (11) is irrelevant.

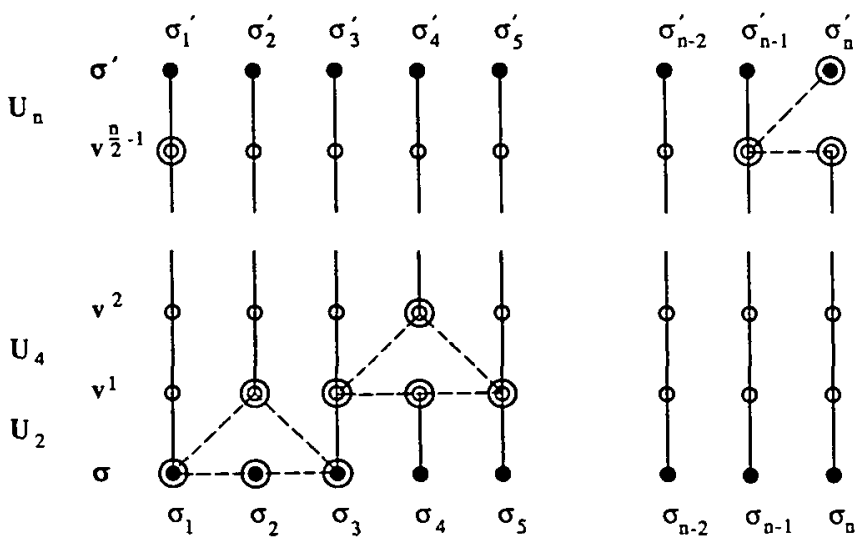

FIGURE 3 
In conclusion, for the square lattice drawn diagonally, we have seen that not only can one write down transfer matrices for the IRF model connecting adjacent rows of interacting spins, but that these transfer matrices themselves can be constructed from local transfer matrices that can be thought of as connecting the faces within a row. This is true also for the row to row transfer matrix.

\subsection{Row to row transfer matrix}

Now consider the arrangement shown in Figure 4 where again we emply periodic boundary conditions with $m$ rows and $n$ columns. The combined Boltzmann weight of the faces shown is

$$
\mathbf{S}\left(\boldsymbol{\sigma} \mid \boldsymbol{\sigma}^{\prime}\right)=\prod_{j=1}^{n} w\left(\boldsymbol{\sigma}_{\jmath}, \boldsymbol{\sigma}_{j+1}, \sigma_{j}^{\prime}, \boldsymbol{\sigma}_{j+1}^{\prime}\right) .
$$

Here $\mathbf{S}$ is $2^{n} \times 2^{n}$ and the partition function (2) is given by

$$
\mathbf{Z}_{N}=\operatorname{Trace}(\mathbf{S})^{m} \text {. }
$$

To factor $\mathbf{S}$ we introduce $n-1$ sets of auxiliary spin variables, each containing $n+2$ elements [11]. Denote these by

$$
\mathbf{u}^{p}=\left\{u_{1}^{p}, \ldots, u_{n+2}^{p}\right\},
$$

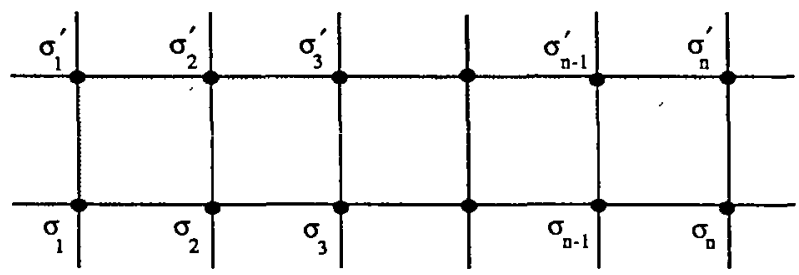

Figure 4

R $\begin{aligned} & \sigma^{\prime} \\ & \mathbf{u}^{\mathrm{n}-1} \oint\end{aligned}$

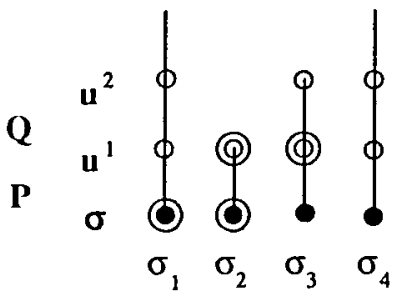

(
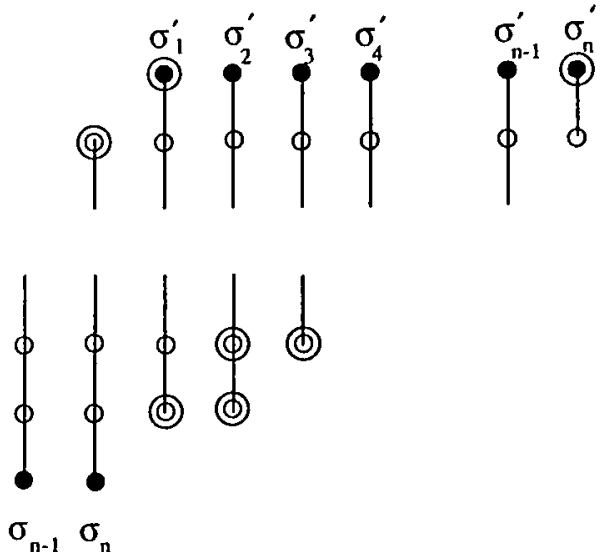

FIGURE 5 
where now $p=1, \ldots, n-1$. Here we draw the hierarchy of interacting spins as in Figure 5. Again we consider it in conjunction with the corresponding row of faces in Figure 4. We can immediately write down the following sum rule

$$
\mathbf{S}\left(\boldsymbol{\sigma} \mid \boldsymbol{\sigma}^{\prime}\right)=\sum_{\left\{\mathbf{u}^{\mathcal{P}}\right\}} \mathbf{P}\left(\boldsymbol{\sigma} \mid \mathbf{u}^{1}\right) \mathbf{Q}\left(\mathbf{u}^{1} \mid \mathbf{u}^{2}\right) \cdots \mathbf{Q}\left(\mathbf{u}^{n-2} \mid \mathbf{u}^{n-1}\right) \mathbf{R}\left(\mathbf{u}^{n-1} \mid \boldsymbol{\sigma}^{\prime}\right),
$$

where

$$
\begin{gathered}
\mathbf{P}\left(\sigma \mid \mathbf{u}^{1}\right)=\delta\left(\sigma_{1}, u_{1}^{1}\right) \cdots \delta\left(\sigma_{n}, u_{n}^{1}\right) w\left(\sigma_{1}, \sigma_{2}, u_{n+1}^{1}, u_{n+2}^{1}\right), \\
\mathbf{Q}\left(\mathbf{u}^{p} \mid \mathbf{u}^{p+1}\right)=\delta\left(u_{1}^{p}, u^{p+1}\right) \delta\left(u_{3}^{p}, u_{2}^{p+1}\right) \\
\cdots \delta\left(u_{n+2}^{p}, u_{n+1}^{p+1}\right) w\left(u_{2}^{p}, u_{3}^{p}, u_{n+1}^{p+1}, u_{n+2}^{p+1}\right), \\
\mathbf{R}\left(\mathbf{u}^{n-1} \mid \sigma^{\prime}\right)=\delta\left(u_{3}^{n-1}, \sigma_{1}^{\prime}\right) \cdots \delta\left(u_{n+2}^{n-1}, \sigma_{n}^{\prime}\right) w\left(u_{1}^{n-1}, u_{2}^{n-1}, \sigma_{1}^{\prime}, \sigma_{n}^{\prime}\right) .
\end{gathered}
$$

Finally we write

$$
\mathbf{S}=\mathbf{P Q}^{\mathbf{n}-2} \mathbf{R}
$$

where $\mathbf{P}$ is $2^{n} \times 2^{n+2}, \mathbf{Q}$ is $2^{n+2} \times 2^{n+2}$ and $\mathbf{R}$ is $2^{n+2} \times 2^{n}$.

Note that the factors $U_{t}$ in the diagonal to diagonal transfer matrix are each different, whereas for the row to row transfer matrix there are $n$ - 2 identical matrices $\mathbf{Q}$. Of the two factorizations for the IRF model, (20) is the more favourable for the numerical evaluation of leading eigenvalues. Nightingale ([9], [10]) used this factorization to compute the leading eigenvalues of the transfer matrix for strips of width up to $n=16$. He was thus able to sucessfully estimate the bulk critical behaviour for the various special cases of the IRF model that are of physical interest.

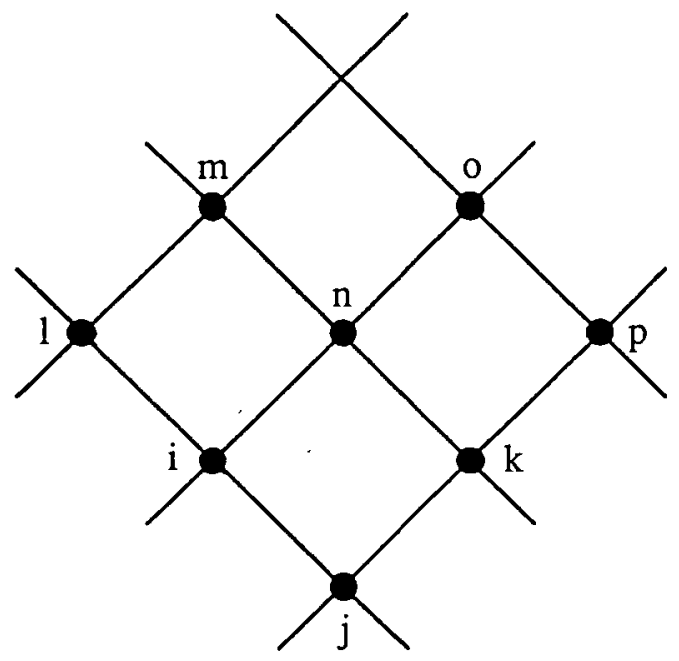

FIGURE 6 


\section{Interactions round a wedge}

To include third nearest neighbour interactions we need to construct a diagonal to diagonal transfer matrix. Consider then a typical wedge $i, j, k, l, m, n, o, p$ shown in Figure 6 with associated Boltzmann weight factor $w\left(\sigma_{i}, \sigma_{j}, \sigma_{k}, \sigma_{l}, \sigma_{m}, \sigma_{n}, \sigma_{o}, \sigma_{p}\right)$. The transfer matrix between spin sets $\sigma$ and $\sigma^{\prime}$ in Figure 7 can then be written as a product of the Boltzmann weights of the (overlapping) wod $\mathfrak{g} c \hat{s}$

$$
\mathbf{T}\left(\boldsymbol{\sigma} \mid \boldsymbol{\sigma}^{\prime}\right)=\prod_{j=1}^{n / 2} w\left(\boldsymbol{\sigma}_{2 j-1}, \sigma_{2 j}, \sigma_{2 j+1}, \sigma_{2 j-2}^{\prime}, \boldsymbol{\sigma}_{2 j-1}^{\prime}, \sigma_{2 j}^{\prime}, \boldsymbol{\sigma}_{2 j+1}^{\prime}, \sigma_{2 j+2}^{\prime}\right) .
$$

Again we impose periodic boundary conditions with $m$ diagonal rows and $n$ columns. The partition function ( 2 ) becomes

$$
\mathbf{Z}_{N}=\operatorname{Trace}(\mathbf{T})^{m}
$$

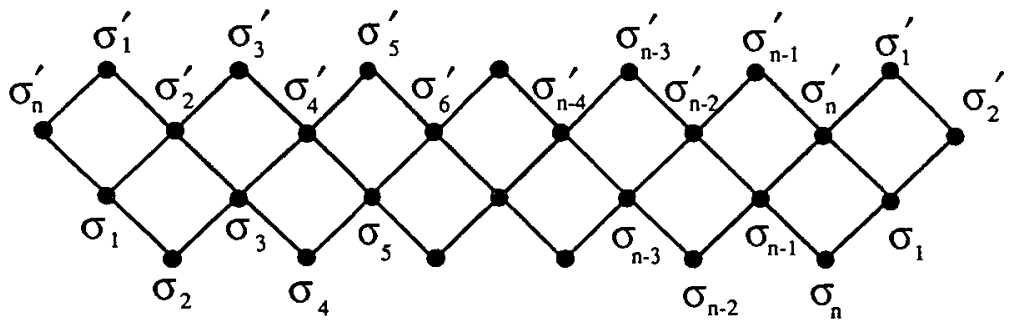

FIGURE 7

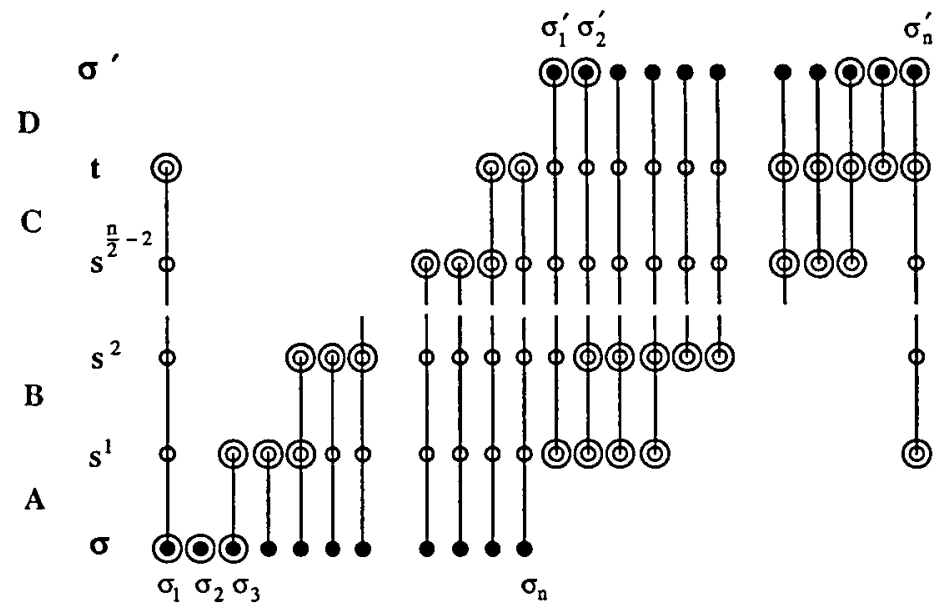

FIGURE 8 
In this case Figure 8 suggests introducing

$$
\begin{aligned}
\mathbf{s}^{p} & =\left\{s_{1}^{p}, \ldots, s_{n+4}^{p}\right\}, \\
\mathbf{t} & =\left\{t_{1}, \ldots, t_{n+3}\right\},
\end{aligned}
$$

with $p=1, \ldots, n / 2-2$. Equation (21) can then be written as

$$
\mathbf{T}\left(\boldsymbol{\sigma} \mid \boldsymbol{\sigma}^{\prime}\right)=\sum_{\left\{\mathbf{s}^{p}\right\}, \mathbf{t}} \mathbf{A}\left(\boldsymbol{\sigma} \mid \mathbf{s}^{1}\right) \mathbf{B}\left(\mathbf{s}^{1} \mid \mathbf{s}^{2}\right) \cdots \mathbf{C}\left(\mathbf{s}^{n / 2-2} \mid \mathbf{t}\right) \mathbf{D}\left(\mathbf{t} \mid \boldsymbol{\sigma}^{\prime}\right),
$$

where

$$
\begin{aligned}
& \mathbf{A}\left(\sigma \mid \mathbf{s}^{1}\right)=\delta(\left.\sigma_{1}, s_{1}^{1}\right) \delta\left(\sigma_{3}, s_{2}^{1}\right) \\
& \cdots \delta\left(\sigma_{n}, s_{n-1}^{1}\right) w\left(\sigma_{1}, \sigma_{2}, \sigma_{3}, s_{n+4}^{1}, s_{n}^{1}, s_{n+1}^{1}, s_{n+2}^{1}, s_{n+3}^{1}\right) \\
& \mathbf{B}\left(\mathbf{s}^{p} \mid \mathbf{s}^{q}\right)=\delta\left(s_{1}^{p}, s_{1}^{q}\right) \delta\left(s_{4}^{p}, s_{2}^{q}\right) \cdots \delta\left(s_{n+3}^{p}, s_{n+1}^{q}\right) \\
& \\
& \cdot \delta\left(s_{n+4}^{p}, s_{n+4}^{q}\right) w\left(s_{2}^{p}, s_{3}^{p}, s_{4}^{p}, s_{n-1}^{q}, s_{n}^{q}, s_{n+1}^{q}, s_{n+2}^{q}, s_{n+3}^{q}\right) \\
& \mathbf{C}\left(\mathbf{s}^{r} \mid \mathbf{t}\right)=\delta\left(s_{1}^{r}, t_{1}\right) \delta\left(s_{4}^{r}, t_{2}\right) \cdots \delta\left(s_{n+3}^{r}, t_{n+1}\right) \\
& \\
& \cdot \delta\left(s_{n+4}^{r}, t_{n+3}\right) w\left(s_{2}^{r}, s_{3}^{r}, s_{4}^{r}, t_{n-1}, t_{n}, t_{n+1}, t_{n+2}, t_{n+3}\right) \\
& \mathbf{D}\left(t \mid \sigma^{\prime}\right)=\delta\left(t_{4}, \sigma_{1}^{\prime}\right) \cdots \delta\left(t_{n+3}, \sigma_{n}^{\prime}\right) w\left(t_{2}, t_{3}, t_{1}, \sigma_{n-2}^{\prime}, \sigma_{n-1}^{\prime}, \sigma_{n}^{\prime}, \sigma_{1}^{\prime}, \sigma_{2}^{\prime}\right),
\end{aligned}
$$

in which $q=p+1$ and $r=n / 2-2$. The final result is

$$
\mathbf{T}=\mathbf{A B}^{n / 2-3} \mathbf{C D} \text {. }
$$

\subsection{Structure of A,B,C and D}

Nightingale [10] has given the explicit structure of the factors $\mathbf{P}, \mathbf{Q}$ and $\mathbf{R}$, defined in (17), (18) and (19), of the row to row transfer matrix for the IRF model. Here we consider the factors A, B, C and D of the more general model. The structure of these matrices can be readily written down once an ordering of the possible configurations is decided upon. Most convenient for numerical purposes is the unique identification of a given spin configuration with the binary representation of an integer. This ordering of states, along with the labelling scheme adopted for the Boltzmann weights, is outlined in Appendix A where we assume the model possess spin reversal symmetry, i.e., the Boltzmann weights remain unchanged on reversing the sign of the spins.

The transfer matrix $\mathbf{T}$ can be written in block form

$$
\mathbf{T}=\left(\begin{array}{ll}
\mathbf{T}_{1} & \mathbf{T}_{2} \\
\mathbf{T}_{2} & \mathbf{T}_{1}
\end{array}\right),
$$


where both $T_{1}$ and $T_{2}$ are square matrices of order $2^{n-1}$. In this representation the $2^{n} \times 2^{n+4}$ matrix $A$, defined via equation (25), assumes the form

$$
\mathbf{A}=\left(\begin{array}{cc}
\mathbf{A}_{1} & \mathbf{0} \\
\mathbf{0} & \mathbf{A}_{1}
\end{array}\right)
$$

with

$$
A_{1}=\left(\begin{array}{cc}
i_{n-3} \otimes \bar{a}_{0} & \hat{0} \\
0 & 1_{n-3} \otimes a_{1} \\
1_{n-3} \otimes a_{2} & 0 \\
0 & 1_{n-3} \otimes a_{3}
\end{array}\right)
$$

Here $\mathbf{1}_{m}$ is the $2^{m} \times 2^{m}$ identity matrix, $\mathbf{a}_{0}, \mathbf{a}_{1}, \mathbf{a}_{2}$ and $\mathbf{a}_{3}$ are row matrices of length 32 defined as

$$
\mathbf{a}_{\imath}=\left(w_{t, 0} w_{i, 16} w_{t, 1} w_{i, 17} \cdots w_{t, 15} w_{t, 31}\right) .
$$

The $2^{n+4} \times 2^{n+4}$ matrix $B$, defined via equation (26), assumes the form

$$
\mathbf{B}=\left(\begin{array}{cc}
\mathbf{B}_{1} & \mathbf{0} \\
\mathbf{0} & \mathbf{B}_{1}
\end{array}\right)
$$

with

$$
B_{1}=\left(\begin{array}{cc}
1_{n-4} \otimes b_{0} & 0 \\
0 & 1_{n-4} \otimes b_{1} \\
1_{n-4} \otimes b_{2} & 0 \\
0 & 1_{n-4} \otimes b_{3} \\
1_{n-4} \otimes b_{4} & 0 \\
0 & 1_{n-4} \otimes b_{5} \\
1_{n-4} \otimes b_{6} & 0 \\
0 & 1_{n-4} \otimes b_{7}
\end{array}\right) .
$$

Each of the $16 \times 64$ matrices $b_{\text {, }}$ is defined as

$$
\mathbf{b}_{i}=\left(\begin{array}{cc}
w_{i, 0} 0 w_{i, 1} 0 w_{i, 2} 0 w_{i, 3} 0 & 0 \\
0 w_{i, 0} 0 w_{i, 1} 0 w a_{t, 2} 0 w_{i, 3} & 0 \\
\mathbf{0} & w_{i, 28} 0 w_{i, 29} 0 w_{i, 30} 0 w_{i, 31} 0 \\
& 0 w_{i, 28} 0 w_{i, 29} 0 w_{i, 30} 0 w_{i, 31}
\end{array}\right) .
$$

The $2^{n+4} \times 2^{n+3}$ matrix $C$, defined via equation (27), assumes the form

$$
C=\left(\begin{array}{cc}
C_{1} & 0 \\
0 & C_{1}
\end{array}\right)
$$


with

$$
C_{1}=\left(\begin{array}{cc}
1_{n-4} \otimes c_{0} & 0 \\
0 & 1_{n-4} \otimes c_{1} \\
1_{n-4} \otimes c_{2} & 0 \\
0 & 1_{n-4} \otimes c_{3} \\
1_{n-4} \otimes c_{4} & 0 \\
0 & 1_{n-4} \otimes c_{5} \\
1_{n-4} \otimes c_{6} & 0 \\
0 & 1_{n-4} \otimes c_{7}
\end{array}\right),
$$

where the $16 \times 32$ matrices $c_{l}$ are defined by

$$
\mathbf{c}_{\imath}=\left(\begin{array}{cccc}
w_{\imath, 0} & 0 & w_{1,2} 0 & 0 \\
0 w_{i, 1} & 0 & w_{i, 3} & \\
0 & w_{i, 28} 0 w_{i, 30} 0 \\
0 & 0 w_{i, 29} 0 w_{i, 31}
\end{array}\right) .
$$

Finally, the $2^{n+3} \times 2^{n}$ matrix $\mathbf{D}$, defined via equation (28) can be seen to take the form

$$
\mathbf{D}=\left(\begin{array}{ll}
\mathbf{D}_{1} & \mathbf{D}_{2} \\
\mathbf{D}_{2} & \mathbf{D}_{1}
\end{array}\right)
$$

in which

$$
\mathbf{D}_{1}=\left(\begin{array}{cc}
\mathbf{1}_{n-5} \otimes \mathbf{e}_{0} & 0 \\
\mathbf{0} & \mathbf{1}_{n-5} \otimes \mathbf{f}_{0} \\
\mathbf{0} & \mathbf{0} \\
\mathbf{0} & \mathbf{0} \\
\mathbf{1}_{n-5} \otimes \mathbf{e}_{2} & \mathbf{0} \\
\mathbf{0} & \mathbf{1}_{n-5} \otimes \mathbf{f}_{2} \\
\mathbf{0} & \mathbf{0} \\
\mathbf{0} & \mathbf{0} \\
\mathbf{1}_{n-5} \otimes \mathbf{e}_{4} & 0 \\
\mathbf{0} & \mathbf{1}_{n-5} \otimes \mathbf{f}_{4} \\
\mathbf{0} & \mathbf{0} \\
\mathbf{0} & \mathbf{0} \\
\mathbf{1}_{n-5} \otimes \mathbf{e}_{6} & \mathbf{0} \\
\mathbf{0} & \mathbf{1}_{n-5} \otimes \mathbf{f}_{6} \\
\mathbf{0} & 0 \\
\mathbf{0} & 0
\end{array}\right)
$$


and

$$
\mathbf{D}_{2}=\left(\begin{array}{cc}
\mathbf{0} & \mathbf{0} \\
\mathbf{0} & \mathbf{0} \\
\mathbf{0} & \mathbf{1}_{n-5}^{A} \otimes \mathrm{g}_{0} \\
\mathbf{1}_{n-5}^{A} \otimes \mathbf{h}_{0} & \mathbf{0} \\
\mathbf{0} & \mathbf{0} \\
\mathbf{0} & \mathbf{0} \\
\mathbf{0} & \mathbf{1}_{n-5}^{A} \otimes \mathrm{g}_{2} \\
\mathbf{1}_{n-5}^{A} \otimes \mathbf{h}_{2} & \mathbf{0} \\
\mathbf{0} & \mathbf{0} \\
\mathbf{0} & \mathbf{0} \\
\mathbf{0} & \mathbf{1}_{n-5}^{A} \otimes \mathbf{g}_{4} \\
\mathbf{1}_{n-5}^{A} \otimes \mathbf{h}_{4} & \mathbf{0} \\
\mathbf{0} & \mathbf{0} \\
\mathbf{0} & \mathbf{0} \\
\mathbf{0} & \mathbf{1}_{n-5}^{A} \otimes \mathrm{g}_{6} \\
\mathbf{1}_{n-5}^{A} \otimes \mathbf{h}_{6} & \mathbf{0}
\end{array}\right)
$$

where we further define $\mathbf{1}_{m}^{A}$ as the $2^{m} \times 2^{m}$ matrix in which all elements, except those along the anti-diagonal, vanish. The $8 \times 8$ matrices $\mathbf{e}_{t}, \mathbf{f}_{\imath}, \mathbf{g}_{t}$ and $\mathbf{h}_{t}$ are defined by

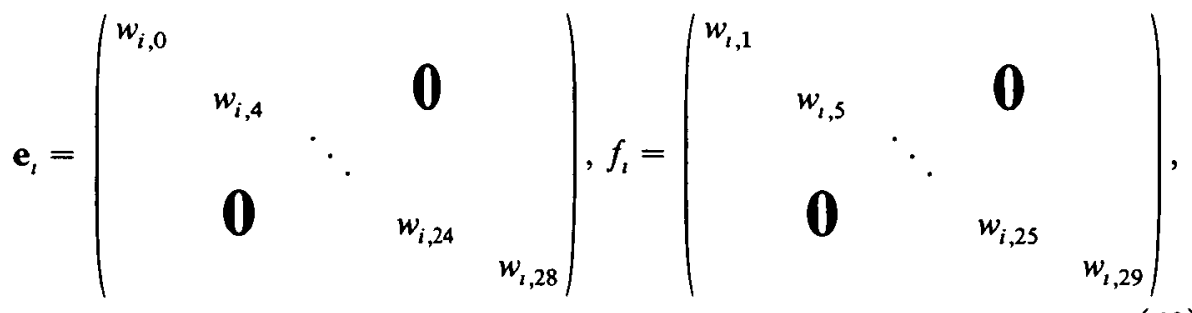

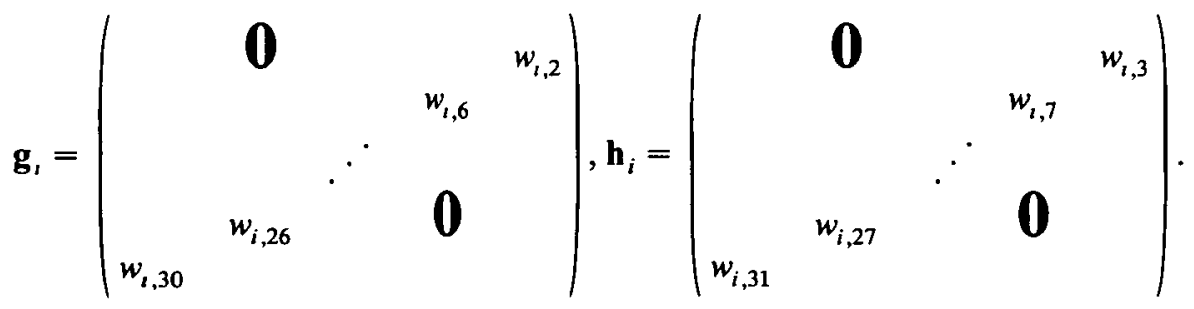


As for Nightingale's factorization of the IRF model, the matrices $\mathbf{T}_{1}$ and $\mathbf{T}_{2}$ can also be factored. Inserting the block forms (31), (34), (37) and (40) into equation (29) and comparing the result with (30) leads to

$$
\begin{aligned}
& \mathbf{T}_{1}=\mathbf{A}_{1} \mathbf{B}_{1}^{n / 2-3} \mathbf{C}_{1} \mathbf{D}_{1}, \\
& \mathbf{T}_{2}=\mathbf{A}_{1} \mathbf{B}_{1}^{n / 2-3} \mathbf{C}_{1} \mathbf{D}_{2},
\end{aligned}
$$

which form the starting point for the leading eigenvalue computations [2].

For numerical purposes, Nightingale [10] has emphasized the significant reduction in the number of elementary arithmetic operations resulting from the use of sparse matrix representations in eigenvalue computations. To see this, consider the direct calculation of $T_{1} \varphi$ from the vector $\varphi$. This requires $2^{n-1} \times 2^{n-1}$ multiplications. When the factorization given in equation (45) is used (by successively generating $\mathbf{D}_{1} \varphi, \mathbf{C}_{1} \mathbf{D}_{1} \varphi$, etc.), the number of multiplications needed can be reduced to that of the number of non-zero matrix elements appearing in the sparse factors. From equations (32), (35), (38), (41) and (45) this number is seen to be $(32 n-124) \times 2^{n-1}$.

\section{Summary and conclusion}

For each of the cases considered the formulation of the transfer matrix as a product of sparse factors has been achieved by viewing the auxiliary spin sets required as a hierarchy of interacting spins. The majority of these spins interact through delta functions, giving rise to the sparse nature of the factors. Each factor of the transfer matrix can be thought of as arising from the fundamental units (faces or wedges) in a given row. The use of the sparse matrix representation leads to a significant reduction in the number of elementary operations required in computing the leading eigenvalues of the transfer matrix.

The prime motivation in seeking the factorization (29) for the general IRW model has been to study [2] the critical behaviour of a two-dimensional Ising model containing isotropic first and third nearest neighbour interactions. Even though the dominant factor $\mathbf{B}$ in the factorization (29) for the transfer matrix of the general model is sixteen times larger than the corresponding matrix $\mathbf{Q}$ in equation (20) for the IRF model, leading eigenvalues have been computed [2] for strips of width up to and including $n=16$.

One can also achieve a sparse matrix factorization with the original helical or screw boundary conditions of Kramers and Wannier [8]. Domb [5] showed that the transfer matrix, T, for $n$ such columns could be written in the form

$$
\mathbf{T}=\mathbf{A}^{n-1} \mathbf{B}
$$

and pointed out that $B$ corresponds to an end effect which, at least on physical grounds, becomes neglibible as $n \rightarrow \infty$. (This T, $\mathbf{A}$ and $\mathbf{B}$ are not be confused with those of the previous section.) In this spirit Gartenhaus [7] and Fuchs and 
Gartenhaus [6] have considered the extent to which the matrix $\mathbf{A}$ is capable of yielding the critical properties of the system under consideration. When combined with the techniques of finite-size scaling, the scheme was seen to be effective.

For the more general models considered in this paper, the matrices $\mathbf{P}$ and $\mathbf{R}$ for the IRF model and matrices $A, C$ and $\mathbf{D}$ for the IRW model can all be considered as end effects arising from the periodic boundary conditions. To what extent the utilization of the matrices $\mathbf{Q}$ and $\mathbf{B}$ in any approximation scheme for larger lattice sizes would be successful is clearly a question for further consideration.

\section{Acknowledgements}

I am grateful to the Commonwealth Department of Education for receipt of a Commonwealth Postgraduate Research Award. I also wish to thank Professor M. N. Barber for helpful conversations and advice.

\section{Appendix A: Ordering of states}

Invoke the one to one correspondence between an $\mathrm{N}$-dimensional spin set $\sigma=\left\{\sigma_{1}, \ldots, \sigma_{N}\right\}$ and the integers $0,1, \ldots, 2^{N}-1$. Define

$$
\begin{gathered}
n_{1}=\frac{1}{2}\left(1-\sigma_{N}\right), \\
n_{2}=\frac{1}{2}\left(1-\sigma_{N-1}\right), \\
\vdots \\
n_{N}=\frac{1}{2}\left(1-\sigma_{1}\right),
\end{gathered}
$$

with

$$
p(\sigma)=\sum_{i=1}^{N} n_{i} 2^{i-1} .
$$

Order the states according to spin reversal:

$$
q(\sigma)= \begin{cases}p(\sigma) & \text { if } p(\sigma) \leqslant 2^{N-1}-1 \\ p(-\sigma)+2^{N-1} & \text { otherwise }\end{cases}
$$

For example, for $N=2$ we order the states as,,,+++----+ :

$\begin{array}{ccc}\sigma & p(\sigma) & q(\sigma) \\ ++ & 0 & 0 \\ +- & 1 & 1 \\ -- & 3 & 2 \\ -+ & 2 & 3\end{array}$.

Label the $2^{8}=256$ Boltzmann weights as

$$
w_{r, s}=w\left(\sigma_{i}, \sigma_{j}, \sigma_{k}, \sigma_{l}, \sigma_{m}, \sigma_{n}, \sigma_{o}, \sigma_{p}\right)
$$


with

$$
\begin{aligned}
& r=p\left(\sigma_{i}, \sigma_{j}, \sigma_{k}\right), \\
& s=p\left(\sigma_{l}, \sigma_{m}, \sigma_{n}, \sigma_{o}, \sigma_{p}\right) .
\end{aligned}
$$

For example $w_{0,0}=w_{7,31}$ when spin reversal symmetry is allowed, though this reduction in notation has not been used explicitly in writing down the structure of the factors $\mathbf{A}, \mathbf{B}, \mathbf{C}$ and $\mathbf{D}$.

\section{References}

[1] M. N. Barber, Finite-size scaling, in Phase Transitions and Critical Phenomena, Vol. 8 (eds. C. Domb and J. L. Lebowitz), (Academic Press, London, 1983), 145-266.

[2] M. T. Batchelor, J. Oitmaa and M. N. Barber, A finite lattice study of the critical behaviour of the two-dimensional biaxial next nearest neighbour Ising model (to appear).

[3] R. J. Baxter, Exactly solved models, in Fundamental Problems in Statistical Mechanics V (ed. E. G. D. Cohen), (North Holland, Amsterdam, 1980), 109-141.

[4] R. J. Baxter, Exactly Solved Models in Statistical Mechanics (Academic Press, London, 1982).

[5] C. Domb, Order-disorder statistics. I, Proc. Roy. Soc. London Ser. A 196 (1949), 36-50.

[6] N. H. Fuchs and S. Gartenhaus, Sparse-matrix analysis of spin-1/2 Ising systems, Phys. Rev. B 31 (1985), 7261-7273.

[7] S. Gartenhaus, Approximation methods for spin-1/2 Ising models, Phys. Rev. B 27 (1983), 1698-1718.

[8] H. A. Kramers and G. H. Wannier, Statistics of the two-dimensional ferromagnet. I, Phys. Rev. 60 (1941), 252-262.

[9] M. P. Nightingale, Non-universality for Ising-like spin systems, Phys. Lett. A 59 (1977), 486-488.

[10] M. P. Nightingale, Phenomenological renormalization group theory, Proc. K. Ned. Akad. Wet. 82 (1979), 235-291.

[11] M. P. Nightingale, Finite-size scaling and phenomenological renormalization, J. Appl. Phys. 53 (1982), 7927-7932. 